\title{
Influence of deposition conditions on morphological, structural, optical and electro-physical properties of ZnSe films obtained by close-spaced vacuum sublimation
}

\author{
M.M. Ivashchenko ${ }^{\text {a,* }}$, I.P. Buryk ${ }^{\text {a }}$, A.S. Opanasyuk ${ }^{b}$, D. Nam ${ }^{c}$, H. Cheong ${ }^{c}$, \\ Ja.G. Vaziev ${ }^{\mathrm{b}}$, V.V. Bibyk ${ }^{\mathrm{a}}$ \\ ${ }^{a}$ Konotop Institute, Sumy State University, 24 Myru Ave., Konotop 41600, Ukraine \\ b Sumy State University, 2 Rimsky-Korsakov Str., Sumy 40007, Ukraine \\ c Sogang University, 35 Baekbeom-ro., Mapo-gu, Seoul 121-742, Republic of Korea
}

\section{A R T I C L E I N F O}

PACS:

61.05.cp

68.37.Hk

78.66.Hf

81.15.Ef

Keywords:

Zinc selenide

Surface morphology

Structure

Optical properties

\begin{abstract}
A B S T R A C T
ZnSe thin films were deposited on well-cleaned glass substrates by the close-spaced vacuum sublimation technique. Various structural, sub-structural and optical properties have been investigated through scanning electron microscopy (SEM), X-ray diffraction (XRD), spectral photometry, Raman spectroscopy and Fourier transform infrared (FTIR) spectroscopy. Electro-physical studies were performed using an ITO/ZnSe/In "sandwich" structure. The correlation between the films structure and their optical and electrophysical properties was estimated. The evaluated films were fine-crystalline, with their grain size depending on the substrate temperature. The optical band gap was estimated through optical measurements and the high optical qualities of the ZnSe films were confirmed by Raman and FTIR analyses.
\end{abstract}

(c) 2015 Elsevier Ltd. All rights reserved.

\section{Introduction}

ZnSe is attracting much interest owing to its unique optical and electrical properties [1,2]. Thin films based on this compound may be used in devices for micro-, acoustic and optoelectronic application [3]. Furthermore, owing to its wide band gap $\left(E_{g}=2.7 \mathrm{eV}\right)$, this compound finds applications as a 'window' layer in tandem solar cells [4], light-emitting diodes [5], thin-film transistors, etc [6].

There are different techniques for fabricating $\mathrm{ZnSe}$ films, such as: chemical bath deposition (CBD) [7], pulsed laser ablation [8], pulsed laser deposition [9], magnetron sputtering [10], thermal evaporation [11], close-spaced evaporation [12], quasi-closed volume [13] and its modification - close-

\footnotetext{
* Corresponding author. Tel.: + 38098 4302003; fax: +380 544725171. E-mail address: m_ivashchenko@ukr.net (M.M. Ivashchenko).
}

spaced vacuum sublimation (CSVS) technique [14]. Venkata Subbaiah et al. [12] described in details the close-spaced evaporation technique. This method allows to obtain the high-quality semiconductor films in optimal growth conditions at rough vacuum. Rusu et al. $[13,15]$ used to produce films quasi-closed volume technique which is based on the deposition space limitation and the chamber geometry. A detailed analysis of this method is presented in Ref. [15]. To obtain high structural and optical quality II-VI films compounds in recent time is widely used the CSVS method [16]. The main distinction between this technique and the previous two is additional quasi-closed volume walls heater. The vapor phase is in a small volume of a limited heated wall, accordingly. The compound's components that arise during dissociation are not removed outside the volume. This allows the films close to stoichiometric [16]. Another advantage of this method is the high vapor pressure during the chalcogenide's condensation that exceeds the residual 
gases pressure in quasi-closed volume in $\sim 10^{3}$ times. It helps to reduce the film pollution by uncontrolled impurities of the vacuum chamber residual atmosphere. Due to the physical and technical features of the evaporator the CSVS method allows to obtain highly-crystalline stoichiometric thin films in conditions close to the thermodynamic equilibrium. A detailed description of the CSVS evaporation device was presented in Ref. [16]. However, except for a few publications [12,13], structural and optical investigations of ZnSe films obtained by a similar method have not been investigated extensively, probably due to the difficulty of controlling the temperature.

The main objective of the paper is the study of the surface morphology, structural, optical and electrophysical properties of ZnSe films deposited by the CSVS technique in order to determine optimal growth conditions of satisfactory for device applications ZnSe films with structural and optical quality.

\section{Experimental details}

ZnSe thin films were deposited onto well-cleaned glass substrates from ZnSe powder by the CSVS technique using VUP-5M (Selmi, Ukraine) vacuum equipment (residual gas pressure was $P=5 \times 10^{-3} \mathrm{~Pa}$ ) under the following evaporation conditions: evaporator temperature $T_{e}=1073 \mathrm{~K}$, substrate temperature $T_{s}=373-873 \mathrm{~K}$, and time of evaporation $t_{1}=1-2 \mathrm{~min}$ (optical and electro-physical investigations) and $t_{2}=7$ min (surface morphology and structural investigations).

The surface morphology of the films was studied using a scanning electron microscope REMMA-103 (Selmi, Ukraine). The average grain size $D$ was determined according to the Jeffries method [17]. The films thickness $l$ was estimated by the interference technique.

The structural study of $\mathrm{ZnSe}$ films was carried out by DRON4-07 X-ray diffractometer with the $\mathrm{Cu}-\mathrm{K}_{\alpha}$ radiation over the range of $20^{\circ} \leq 2 \theta \leq 80^{\circ}$, where $2 \theta$ is the Bragg angle. The diffraction patterns were normalized to the most intense (111) peak of the cubic phase. The phase analysis was performed by comparing of inter-planar distances and relative intensities from the samples and the references (JCPDS: 37-1463 and 5-522). The texture of the films was measured by the Harris method. The lattice parameters were estimated by the Bradley-Jay and Nelson-Riley extrapolation methods [17].

The XRD analysis was also used to determine the coherent scattering domain (CSD) size and micro-stress level $\varepsilon$ of the $\mathrm{ZnSe}$ films. To separate the pure and instrumental contributions of the diffraction line broadening profiles the Cauchy, Gauss approximation and threefold convolution [18] were used.

Optical characterizations of the films were carried out by using a double-beam spectrophotometer in the wavelength range of $\lambda=350-600 \mathrm{~nm}$ at room temperature. Optical transmission $T(\lambda)$ and reflection $R(\lambda)$ spectra were measured. Further spectral distributions of absorption $\alpha$ $(\lambda)$, refraction $n(\lambda)$, extinction coefficients $k(\lambda)$, real and imaginary parts $\varepsilon_{1}(\lambda)$ and $\varepsilon_{2}(\lambda)$ of optical dielectric constants were calculated from the spectra in the range of strong light absorption using the Lambert's equation: $\alpha=-$ $\ln (T) / l$ and using the expressions described in Ref. [17].
Raman spectroscopy measurements were performed at room temperature ( $297 \mathrm{~K}$ ) with a TRIAX 550 (1800 grooves/ $\mathrm{mm}$ ) spectrometer, using as the excitation sources a He-Cd laser with the wavelength of $\lambda=441.6 \mathrm{~nm}$. The dispersed signal was detected with a liquid-nitrogen-cooled chargecoupled-device (CCD) detector array. Each spectrum was accumulated 20 times with an acquisition time of 5 s. Longpass Raman edge filters were used to eliminate Rayleigh scattered light.

Fourier transform infrared (FTIR) spectroscopy was carried out by using an Agilent Cary 630 spectrometer. The FTIR spectra were obtained in the full-refraction regime (Diamand ATR appliance). The measured spectral range was varied in the range of $\nu=400-2500 \mathrm{~cm}^{-1}$.

The electro-physical properties of the ZnSe films were estimated by measuring their dark current-voltage $(I-V)$ curves at room temperature according to the standard methods $[19,20]$. The charge transport mechanism was determined using the DC conduction measurement technique [21,22], which allows calculation of the electrical parameters such as the carriers mobility and concentration and the trap density.

\section{Results and discussion}

\subsection{Surface morphology study}

The film grown at $T_{s}=373 \mathrm{~K}$ (Fig. 1a) exhibits layered island growth. The grain size was $D=120-200 \mathrm{~nm}$. At low $T_{S}$ and high vapor saturation increasing of the crystalline size was almost absent due to the intensive secondary nucleation on the film surface. The grains acquired the equi-axed form, which are approximately spherical. As the substrate temperature is increased from 473 to $873 \mathrm{~K}$ the secondary nucleation probability is decreased, the islands coalescence and continuous films are formed (Fig. 1d). The average grain size increased from $0.8 \mu \mathrm{m}$ (Fig. 1b) to $3-4 \mu \mathrm{m}$ (Fig. 1d) which may be caused by the conditions approaching the thermodynamic equilibrium. It should be noted, that especially grains increasing is observed at $T_{s}=873 \mathrm{~K}$. It may be caused by transition from the molecular to gas-dynamic evaporator - substrate vapor transition. Moreover, changes in the morphology may be described by the growth mechanism changing from layer-wise to columnar (substrate temperature must be more than $1 / 3 \mathrm{ZnSe}$ melting point $T=1793 \mathrm{~K})$. Such growth mechanism is stipulated by the crystalline rapid accretion oriented onto (111) plane parallel to the film substrate. It was formed an axial growth texture [111] perpendicular to the surface, consequently. Similar results were obtained by us in the case of CdSe and $\mathrm{ZnS}$ films in Refs. [22,23]. In contrast on CdSe films [22] at similar deposition conditions $\mathrm{ZnSe}$ films had a smaller crystalline size. It, obviously, may be connected to the bigger $\mathrm{ZnSe}$ melting point value $(1793 \mathrm{~K})$ than CdSe melting point (1531 K).

\subsection{Structural investigation}

XRD patterns of the ZnSe films obtained at different substrate temperatures are shown in Fig. 2. The (111) peak is dominant in all cases which indicates the presence of 

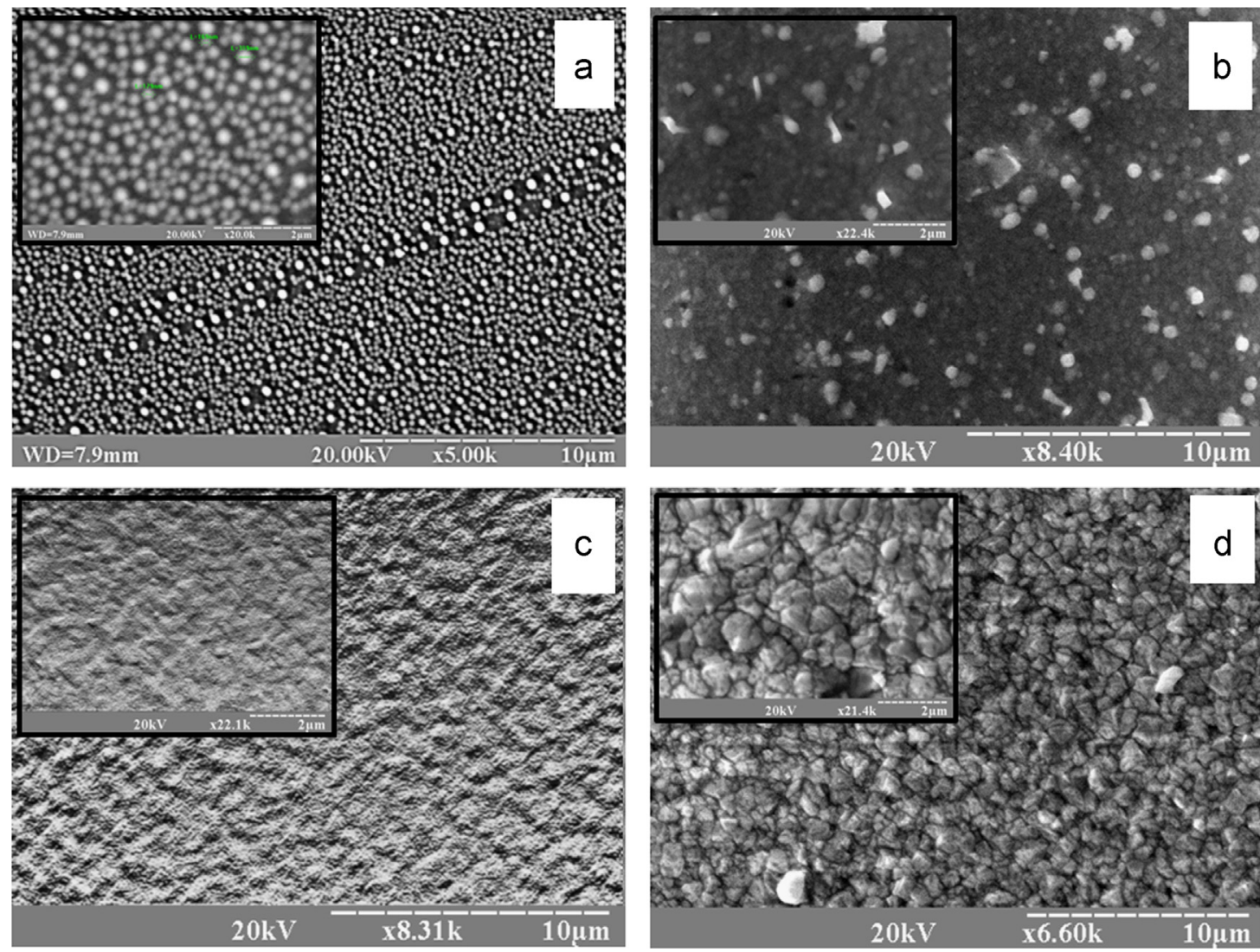

Fig. 1. SEM images of ZnSe films showing surface morphology. $T_{s}$, K: a. 373; b. 473; c. 673; d. 873. Insets higher magnification images.

the [111] films growth texture. This texture is typical for ZnSe films obtained by vacuum methods [24]. In hightemperature samples $\left(T_{s}>473 \mathrm{~K}\right)$ peaks with weak intensities corresponding to reflections from (220), (311) and (222) planes of the cubic (sphalerite) phase were observed (inset of Fig. 2).

A precise determination of the lattice parameters gives an opportunity to study stoichiometric changes of the material, oxidation, presence of impurities, etc. [11]. Fig. 3 shows that the estimates of the lattice parameters by Bradley-Jay and Nelson-Riley approximation methods are similar to each other.

For the cubic phase of ZnSe charge powder $a=$ $0.5666 \mathrm{~nm}$. As the substrate temperature is increased, the lattice parameter changes in the range of $a=0.5652-$ $0.5704 \mathrm{~nm}$. These results agree well with the JCPDS data for bulk ZnSe ( $a=0.5669 \mathrm{~nm})$ [25] and the experimental values obtained for the (111) reflection for $\mathrm{ZnSe}$ thin films deposited by similar methods $a=0.566-0.571 \mathrm{~nm}$ [12].

The inset of Fig. 3 shows the dependence of the orientation factor on the substrate temperature. It should be noted that at $T_{s}=673 \mathrm{~K}$ a decrease of the orientation factor compared to the other results is observed. It may be caused by the growth mechanism changing from layerwise to columnar. A similar effect was observed in the surface morphology.

XRD analysis was also used for investigation of the substructure of ZnSe films by broadening of (111)-(222)

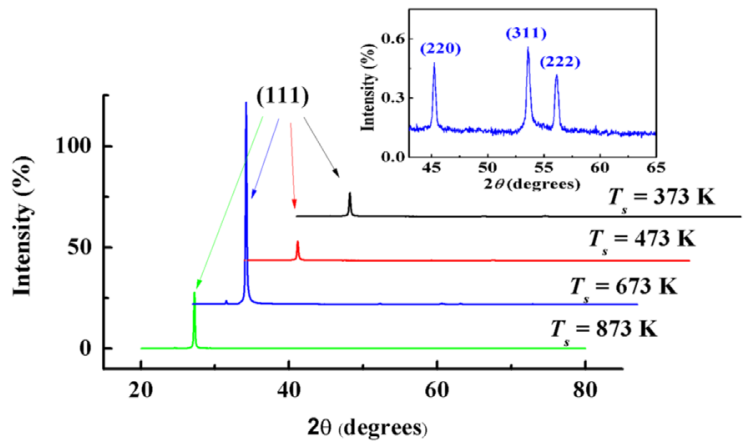

Fig. 2. X-ray diffraction (XRD) patterns of ZnSe films. Inset shows detailed pattern of the film obtained at $T_{s}=673 \mathrm{~K}$.

diffraction peaks. It was estimated that a broadening of the lines corresponds to the sub-grains dispersion, which makes it possible to measure the CSD size $L$ and microstress level $\varepsilon$ of the samples. Fig. 4 presents the dependence of $L$ and $\varepsilon$ on the substrate temperature measured by the Gauss and Cauchy approximations and the threefold convolution. It should be noted that calculated values are agree fairly well with each other. Because the substructure parameters obtained by the last method are the most precise, further discussion of the results will use the values obtained by this method. 


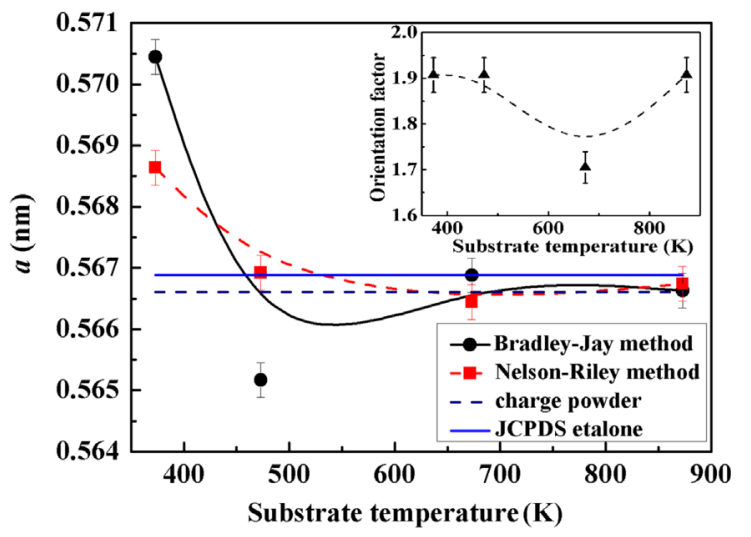

Fig. 3. Substrate temperature dependence of lattice parameter of obtained ZnSe films. Inset shows substrate temperature dependence of the orientation factor.

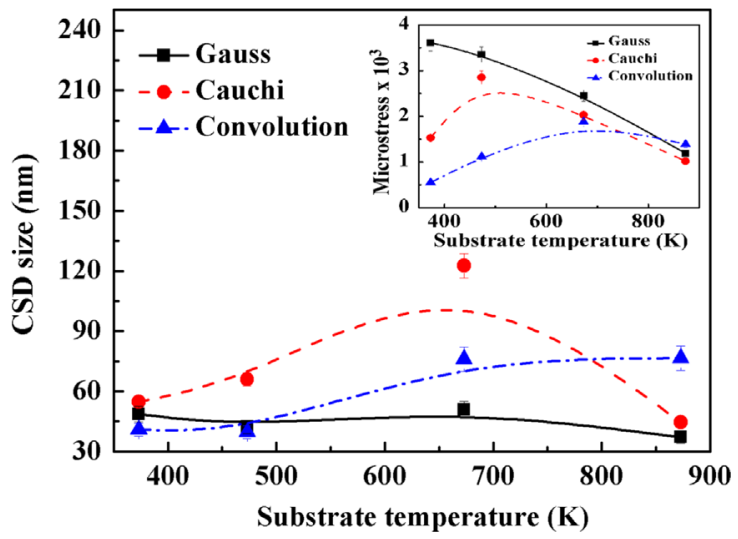

Fig. 4. Substrate temperature dependences of coherent scattering domain (CSD) size and micro-stress (inset) of $\mathrm{ZnSe}$ films.

Table 1

Sub-structure properties of ZnSe thin films.

\begin{tabular}{llllll}
\hline $\begin{array}{l}T_{S} \\
(\mathrm{~K})\end{array}$ & $\begin{array}{l}L \\
(\mathrm{~nm})\end{array}$ & $\varepsilon \times 10^{3}$ & $\begin{array}{l}\rho_{L} \times 10^{-14} \\
\left(\mathrm{lin} / \mathrm{m}^{2}\right)\end{array}$ & $\begin{array}{l}\rho_{\varepsilon} \times 10^{-14} \\
\left(\operatorname{lin} / \mathrm{m}^{2}\right)\end{array}$ & $\begin{array}{l}\rho_{L \varepsilon} \times 10^{-14} \\
\left(\mathrm{lin} / \mathrm{m}^{2}\right)\end{array}$ \\
\hline 373 & 39.70 & 0.55 & 18.00 & 0.12 & 1.10 \\
473 & 39.40 & 1.12 & 19.00 & 0.05 & 0.71 \\
673 & 76.02 & 1.40 & 5.20 & 1.40 & 2.00 \\
873 & 76.50 & 1.05 & 5.10 & 0.76 & 1.50 \\
\hline
\end{tabular}

As we can see in Fig. 4, the CSD size in the direction perpendicular to the (111) plane increases from $L=39.7 \mathrm{~nm}$ to $L=76.5 \mathrm{~nm}$ as $T_{s}$ increases, whereas the micro-stress level $\varepsilon$ reaches a maximum at $T_{s}=673 \mathrm{~K}$ $\left(\varepsilon=1.40 \times 10^{3}\right)$ then decreases to $\varepsilon=1.05 \times 10^{3}$ at $T_{s}=$ $873 \mathrm{~K}$. The calculated sub-structure characteristics of the ZnSe films are summarized in Table 1.

The average dislocation densities which take into account the CSD size $\left(\rho_{L}\right)$, micro-stress level $\left(\rho_{\varepsilon}\right)$ and both cases $\left(\rho_{L \varepsilon}\right)$ are also presented in Table 1 . Corresponding ratios for their determination are presented in Ref. [26]. These values are agreeing well each other. As we can see from Table 1 , ZnSe

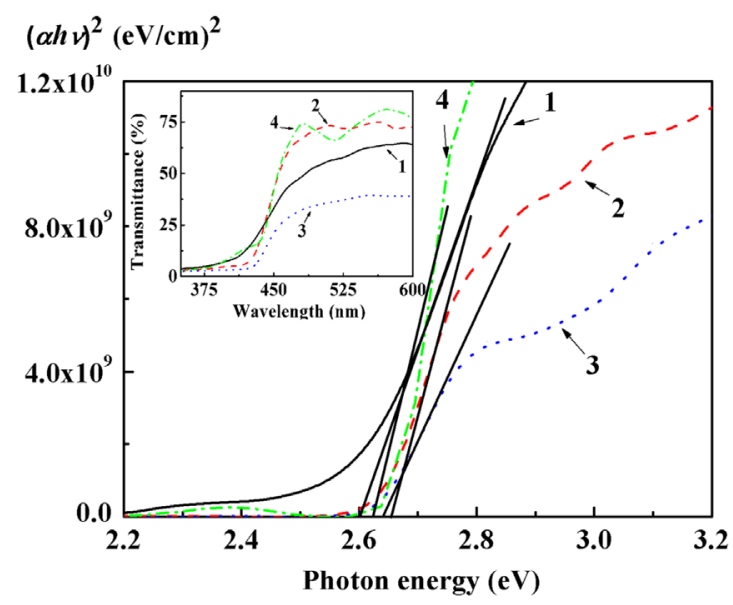

Fig. 5. Band gap values of $\mathrm{ZnSe}$ films. Inset shows corresponding transmittance spectra. $T_{s}$, K: $1.373 ; 2.473 ; 3.673 ; 4.873$.

Table 2

Optical constants of ZnSe films.

\begin{tabular}{llllll}
\hline$T_{s}(\mathrm{~K})$ & $E_{g}(\mathrm{eV})$ & $n$ & $k$ & $\varepsilon_{1}$ & $\varepsilon_{2}$ \\
\hline 373 & 2.78 & 1.35 & $3.72 \times 10^{-4}$ & 1.83 & $1.01 \times 10^{-3}$ \\
473 & 2.75 & 2.81 & $1.23 \times 10^{-4}$ & 7.90 & $0.69 \times 10^{-3}$ \\
673 & 2.68 & 1.61 & $4.47 \times 10^{-4}$ & 2.60 & $1.44 \times 10^{-3}$ \\
873 & 2.70 & 3.10 & $3.38 \times 10^{-4}$ & 9.63 & $2.10 \times 10^{-3}$ \\
\hline
\end{tabular}

films are characterized by dislocations which are in general concentrated on CSD boundaries [27]. Since dislocations act as recombination centers, polycrystalline films may be used as basis layers of devices with improved characteristics.

\subsection{Optical spectroscopy}

The transmission spectra $T(\lambda)$ of the ZnSe films and determination of the optical band gap energy $E_{g}$ are presented in Fig. 5. From the $T(\lambda)$ spectra (inset of Fig. 5) we observed maxima and minima of the intensity which are related to the interference of light in thin layers of chalcogenides. Interference peaks attest to the homogeneity of the investigated areas. To determine $E_{g}$, the linear parts of $(\alpha h \nu)^{2}-h \nu$ graphs were extrapolated to the photon energy axis. The intersepts on the $h \nu$ - axis represented the optical band gap $E_{g}$ of investigated films. The accuracy of $E_{g}$ determination was $\Delta= \pm 0.01 \mathrm{eV}$. Determined band gap values were in the range $E_{g}=2.68-2.78 \mathrm{eV}$. These data are similar to results of the optical investigations of ZnSe films, obtained by thermal evaporation technique $\left(E_{g}=2.60\right.$ $2.64 \mathrm{eV})$ [11], quasi-closed volume $\left(E_{g}=2.62-2.70 \mathrm{eV}\right)[13]$ and to the reference data of bulk $\mathrm{ZnSe}\left(E_{g}=2.70 \mathrm{eV}\right)[1]$ Band gap variety may be caused by the presence of hexagonal phase in $\mathrm{ZnSe}$ films at $T_{s}$ increasing. The optical constants of the $\mathrm{ZnSe}$ films are summarized in Table 2. Such determination was possible owing to the high optical quality of the thin films prepared by the CSVS technique. This suggests that such films may be used as a window layer in CdTe-, CIGS- and CZTS(Se)-based solar cells, green-blue wavelength light-emitted diodes, etc. 


\subsection{Raman spectroscopy investigation}

Fig. 6 shows the Raman spectra of $\mathrm{ZnSe}$ thin films growth at different substrate temperatures. All of the samples show the LO mode of ZnSe at $\sim 250 \mathrm{~cm}^{-1}$, which is close to the well-known frequency for a bulk $\mathrm{ZnSe}$ $252 \mathrm{~cm}^{-1}$ [27].

Moreover, higher order LO modes are shown for at least up to the fourth order. The LO mode is slightly red-shifted relative to the single-crystal $\mathrm{ZnSe}$ case. As the substrate temperature increases, it blue-shifts appreciably (inset of Fig. 6). For $T_{s}=373 \mathrm{~K}$, the $1 \mathrm{LO}$ peak appears at 249 and it shifts to 250 , and $251 \mathrm{~cm}^{-1}$ at substrate temperatures of 473 and $673 \mathrm{~K}$, respectively (Table 3 ). This shift is attributed to the change in the lattice constant caused by the induced strain from the substrate.

The mismatch in the thermal expansion coefficient of the substrate and the $\mathrm{ZnSe}$ thin films would create biaxial strain in the film. The red-shift indicates a tensile strain. Up to $T_{s}=473 \mathrm{~K}$ the shift gets larger which indicates that the induced strain also gets larger. At $T_{s}=673 \mathrm{~K}$ the peak coincides with the $1 \mathrm{LO}$ of bulk $\mathrm{ZnSe}$, which indicates that the strain is relaxed for these films.

\subsection{Fourier transform IR spectroscopy study}

Fourier transform infra-red (FTIR) spectroscopy is an important analysis method to determine functional groups and bonding in semiconductor compounds. The FT-IR spectra of the ZnSe films are shown in Fig. 7.

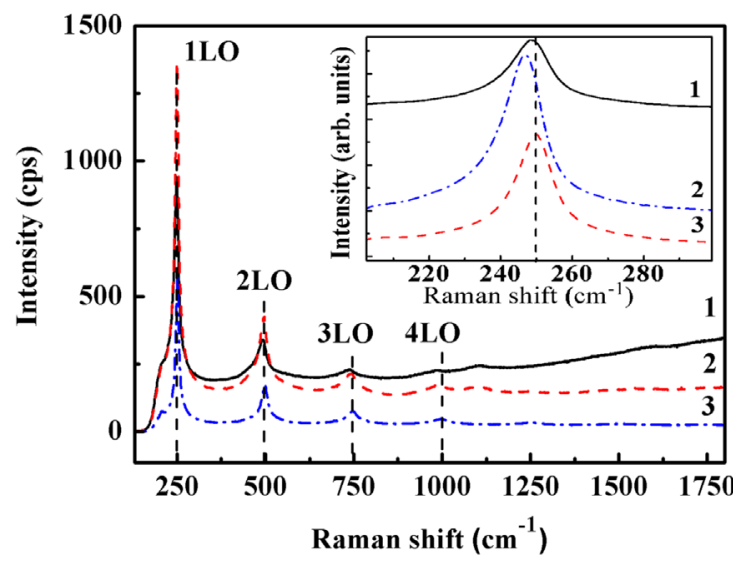

Fig. 6. Raman spectra of ZnSe films. Inset shows a detailed view near the $1 \mathrm{LO}$ peak. $T_{s}, \mathrm{~K}: 1.373 ; 2.473 ; 3.673$.

Table 3

List of Raman peaks frequencies.

\begin{tabular}{llll}
\hline Mode & $T_{s}=373 \mathrm{~K}$ & $T_{s}=473 \mathrm{~K}$ & $T_{s}=673 \mathrm{~K}$ \\
\hline Raman shift & $\nu\left(\mathrm{cm}^{-1}\right)$ & $\nu\left(\mathrm{cm}^{-1}\right)$ & $\nu\left(\mathrm{cm}^{-1}\right)$ \\
1LO & 250 & 249 & 251 \\
2LO & 494 & 497 & 500 \\
3LO & 743 & 746 & 749 \\
4LO & 995 & 995 & 998 \\
\hline
\end{tabular}

At frequencies $627-631 \mathrm{~cm}^{-1}$ a characteristic $\mathrm{Zn}-\mathrm{Se}$ stretching vibration mode is observed whereas a typical $\mathrm{Zn}$-Se bending vibration mode is observed at 726$744 \mathrm{~cm}^{-1}$ [28]. Other peaks are observed at $\sim 850,925$, $1110,1150,1250,1400,1540,1590$ and $2160 \mathrm{~cm}^{-1}$. The assignments of these peaks are summarized in Table 4.

The peaks shift at different substrate temperatures caused by the tensile strains in the ZnSe film due to the substrate material (glass). Similar effects were observed in Raman spectroscopy investigations. Also, higher-frequency peaks at the wave-numbers higher than $1200 \mathrm{~cm}^{-1}$ do not shift much, probably because they are the vibration modes of molecules situated near the film surface. The absence of peaks at wave-numbers higher than $2200 \mathrm{~cm}^{-1}$ indicates the absence of the impurities while ZnSe films were deposited $[30,31]$.

\subsection{Electro-physical investigations}

Fig. 8 shows the $I-V$ curves of ITO/ZnSe/In sandwich structures obtained at different substrate temperatures. In the low-voltage region the conduction mechanism is ohmic ( $U^{1}$ region). In the second voltage region $\left(U^{2}\right)$ trap square law dependence was observed. In the voltage region $U^{3}$ the conduction mechanism changes to the space charge limited current (SCLC) [32]. The increase of the current in the $U^{3}$ region suggests that the dominant trapping levels lie above the Fermi level.

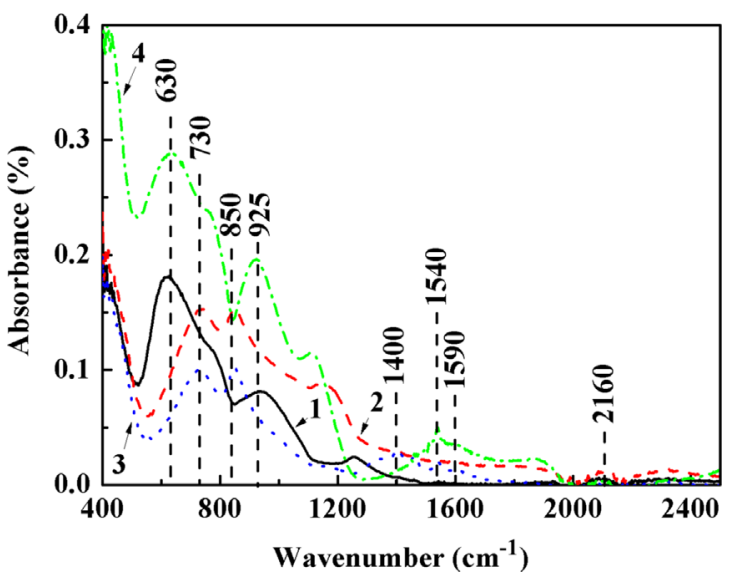

Fig. 7. FTIR spectra of ZnSe films. $T_{s}$, K: 1. 373; 2. 473; 3. 673; 4. 873.

Table 4

FTIR peaks assignments of ZnSe films.

\begin{tabular}{rll}
\hline$\nu\left(\mathrm{cm}^{-1}\right)$ & Assignment & Ref. \\
\hline 850 & Zn-Se stretching vibration mode & {$[27]$} \\
925 & Zn-Se or Se-O stretching vibration mode & {$[28]$} \\
1110 & Zn-Se stretching vibration mode & {$[29]$} \\
1150 & Zn-Se stretching vibration mode & {$[29]$} \\
1250 & C-O bending mode & {$[28]$} \\
1400 & N-NH bending mode & {$[29]$} \\
1540 & C-N bending mode (residual atmosphere) & {$[28]$} \\
1590 & O-H characteristic vibration mode & {$[27]$} \\
2160 & C-O stretching vibration mode & {$[26]$} \\
\hline
\end{tabular}




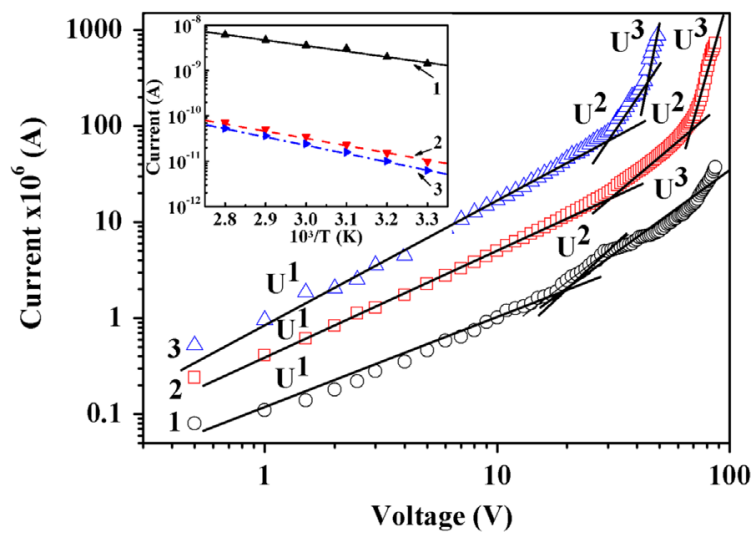

Fig. 8. Current-voltage $(I-V)$ curves of ZnSe films. $T_{s}$, K: $1.673 ; 2.773 ; 3$. 873. Inset shows current vs absolute temperature dependences.

For $n$-type ZnSe, the SCLC density flowing through the film $J$ is obtained from the following expression [19]:

$J=\frac{9}{8} \varepsilon_{s} \mu \theta \frac{U^{2}}{l^{3}}$

where $J$ is the current density flowing through the film; $\varepsilon_{S}$ $=\varepsilon^{\prime} \times \varepsilon_{0}\left(\varepsilon^{\prime}\right.$ is the dielectric constant of the material, and $\varepsilon_{0}$ is a dielectric permittivity in free space); $\theta$ is the Lampert's parameter; $\mu$ is the electron mobility; $U$ is the bias voltage; and $l$ is the film thickness (at $T_{s}=673 \mathrm{~K}$, $l=1.5 \mu \mathrm{m} ; \quad$ at $\quad T_{s}=773 \mathrm{~K}, \quad l=1.8 \mu \mathrm{m} ; \quad$ at $\quad T_{s}=873 \mathrm{~K}$, $l=1.3 \mu \mathrm{m})$.

The value of $N_{t}$ was calculated from the following equation [33]

$V_{T F L}=\frac{q N_{t} l^{2}}{2 \varepsilon_{S}}$

where $q$ is the electronic charge; and $V_{T F L}$ is the trap filled limit voltage.

The depth of the dominant trap level $E_{t}$ below the conduction band edge was calculated by the following ratio [33]

$\theta=\left(\frac{N_{C}}{N_{t}}\right) \exp \left(\frac{-E_{t}}{k T}\right)$,

where $E_{t}\left(N_{t}\right)$ is the ionization energy (concentration of the dominant donor-type trap communication); $T$ is the absolute temperature; $k$ is the Boltzmann constant; and $N_{C}$ is the effective density of states in the conduction band. It was obtained from the relation

$N_{C}=2\left(2 \pi m_{e}^{*} k T / h^{2}\right)^{3 / 2}$,

where $m_{e}^{*}$ is the electron rest mass; and $h$ is the Planck constant.

The results of the electro-physical analyses of the $\mathrm{ZnSe}$ films are presented in Table 5.

In ZnSe films a range of trap levels with the energy depth $E_{t}=0.18-0.31 \mathrm{eV}$ and $N_{t}=1.17 \times 10^{21}-1.68 \times 10^{22} \mathrm{~m}^{-3}$ was revealed. The accuracy of $E_{t}$ determination was no more than $k T(0.026 \mathrm{eV})$ at room temperature. Obtained $E_{t}$ values have some difference each other. It may be caused by the films spatial heterogeneity, i.e. the traps concentration near the substrate is bigger than in film's open surface. It has a good
Table 5

Electro-physical characteristics of ZnSe films.

\begin{tabular}{lllll}
\hline$T_{S}(\mathrm{~K})$ & $V_{T R}(\mathrm{~V})^{\mathrm{a}}$ & $V_{\text {TFL }}(\mathrm{V})$ & $N_{t}\left(\mathrm{~m}^{-3}\right)$ & $E_{t}(\mathrm{eV})$ \\
\hline 673 & 19.54 & 25.60 & $1.17 \times 10^{21}$ & 0.18 \\
773 & 29.69 & 70.63 & $1.373 \times 10^{22}$ & 0.29 \\
873 & 30.69 & 45.07 & $1.680 \times 10^{22}$ & 0.31 \\
\hline
\end{tabular}

${ }^{\text {a }} V_{T R}$ - is the transition voltage between ohmic conduction and SCLS

agreement with structural investigations results which shown about the grain size increase and films quality improvement at $T_{S}$ increasing.

These results suggested exponential trap distribution in all cases of investigated ZnSe films.

\section{Conclusions}

It has been shown that $\mathrm{ZnSe}$ films deposited by the CSVS technique possess a cubic structure. All investigated films have a typical growth texture [111]. Spectral distributions of optical constants were measured, and Raman spectroscopy and FTIR study of the films have been carried out. These results demonstrate high optical quality and structural perfection. Electro-physical investigation have shown that in all cases of investigated ZnSe films three different regions on $I-V$ curves are observed, corresponding to: $U^{1}$ region - ohmic conduction mechanism; $U^{2}$ region - is typical trap square law dependence; and $U^{3}$ region -conduction mechanism based on space charge limited current. The conditions for obtaining single phase, high-textured stoichiometric ZnSe films with good optical and electro-physical characteristics have been determined.

\section{Acknowledgments}

This work is supported by the Ukraine State Agency for the Science, Innovation and Informatization and by the NRF grant funded by the MSIP of Korea (2011-0019204) and by the Ministry of Education and Sciences of Ukraine (Grant no. 0113U000131).

\section{References}

[1] S. Kasap, P. Capper, Springer Handbook of Electronic and Photonic Materials, Springer Science + Business Media Inc, New York, NY, USA, 2006, 1406.

[2] R. Bhargava, Properties of Wide Band Gap II-VI Semiconductors, IEEE/Inspec, London, 1997, 238.

[3] A. Kathalingam, T. Mahalingam, C. Sanjeeviraja, Mater. Chem. Phys. 106 (2007) 215.

[4] R.B. Kale, C.D. Lokhande, Mater. Res. Bull. 39 (2004) 1829.

[5] I. Mora-Sero, V. Munoz, M. Barbe, R. Triboulet, J. Cryst. Growth 197 (1999) 497.

[6] A. Wei, X. Zhao, J. Liu, Y. Zhao, Physica B 410 (2013) 120.

[7] L. Chen, D. Zhang, G. Zhai, J. Zhang, Mater. Chem. Phys. 120 (2010) 456.

[8] T.M. Khan, M. Zakria, M. Amad, R.I. Shakoor, J. Lumin. 147 (2014) 97.

[9] X. Zhang, M. Beres, Z. Ma, S.S. Mao, Appl. Phys. A 114 (2014) 347.

[10] A. Karatay, H.G. Yaglioglu, A. Elmali, M. Parlak, H. Karaagac, Opt. Commun. 285 (2012) 1471.

[11] E. Bacaksiz, S. Aksu, I. Polat, S. Yilmaz, M. Altunbas, J. Alloy. Compd. 487 (2009) 280. 
[12] Y.P. Venkata Subbaiah, P. Prathap, M. Devika, K.T. Ramakrishna Reddy, Physica B 365 (2005) 240.

[13] G.I. Rusu, M.E. Popa, G.G. Rusu, I. Salaoru, Appl. Surf. Sci. 218 (2003) 222.

[14] A.S. Opanasyuk, D.I. Kurbatov, M.M. Ivashchenko, I.Yu. Protsenko, H. Cheong, J. Nano Electron. Phys. 4 (1) (2012) 01024. 3.

[15] M. Rusu, I.I. Nicolaescu, G.G. Rusu, Appl. Phys. A. 70 (2000) 565.

[16] V. Kosyak, A. Opanasyuk, P.M. Bukivskij, Yu.P. Gnatenko, J. Cryst. Growth 312 (2010) 1726.

[17] D. Kurbatov, A. Opanasyuk, H. Khlyap, Phys. Status Solidi A 206 (7) (2009) 1549.

[18] Yu.P. Gnatenko, A.S. Opanasyuk, M.M. Ivashchenko, P.M. Bukivskij, I. O. Faryna, Mater. Sci. Semicon. Process. 26 (2014) 663.

[19] K. Kao, W. Hwang, Electrical Transport in Solids, Pergamon Press, Oxford, UK, 1981, 663.

[20] A.S. Opanasyuk, I.Yu. Protsenko, N.V. Tirkusova, J. Phys. Stud. 4 (2) (2000) 208.

[21] S. Venkatachalam, D. Mangalaraj, S.K. Narayandass, K. Kim, J. Yi, Vacuum 81 (2007) 928.

[22] Yu P. Gnatenko, P.M. Bukivskij, I.O. Faryna, A.S. Opanasyuk, M. M. Ivashchenko, J. Lumin. 146 (2014) 174.

[23] D. Kurbatov, A. Opanasyuk, S. Kshnyakina, V. Melnik, V. Nesprava, Rom. J. Phys. 55 (1-2) (2010) 213.
[24] G.I. Rusu, M. Diciu, C. Pirghie, E.M. Popa, Appl. Surf. Sci. 253 (2007) 9500.

[25] Selected Powder Diffraction Data for Education and Training (Search Manual and Data Cards), USA, 1988.

[26] A.M.E. Raj, S.M. Delphine, S. Sanjeeviraja, M. Jayachandran, Physica B 405 (2010) 2485

[27] D. Schmeltzer, R. Beserman, J. Phys. C: Solid State Phys. 15 (1982) 4173.

[28] M. Arslan, A. Maqsood, A. Mahmood, A. Iqbal, Mater. Sci. Semicon. Process. 16 (2013) 1797.

[29] T.J. Khan, M.F. Mehmood, A. Shah, Q. Raza, A. Iqbal, U. Aziz, Thin Solid Films 519 (2011) 5971.

[30] K. Nakamoto, Infrared and Raman Spectra of Inorganic and Coordination Compounds. Part A. Theory and Applications in Inorganic Chemistry, sixth ed. Wiley, Weinheim, Germany, 2009, 432.

[31] C. Mehta, G.S.S. Saini, J.M. Abbas, S.K. Tripathi, Appl. Surf. Sci. 256 (2009) 608.

[32] D. Kurbatov, V. Kosyak, A. Opanasyuk, V. Melnik, Physica B 404 (2009) 5002.

[33] S. Venkatachalam, D. Mangalaraj, Sa.K. Narayandass, Physica B 393 (2007) 47. 